\title{
Recurrent urinary tract infection: Rates and prescription patterns in the antibiotic stewardship era
}

\author{
Duane R. Hickling, MD, MSCI, FRCSC
}

Division of Urology, Department of Surgery, The Ottawa Hospital, University of Ottawa, Ottawa, ON, Canada

Cite as: Hickling DR. Recurrent urinary tract infection: Rates and prescription patterns in the antibiotic stewardship era. Can Urol Assoc J 2021;15(12):405-6. http://dx.doi.org/10.5489/cuaj.7697

See related article on page 397

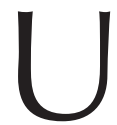
rinary tract infection (UTI) is one of the most common human bacterial infections and is associated with tremendous morbidity in quality of life and economic measures. Recurrent UTIs (rUTIs), defined as three documented UTIs within 12 months or two documented UTIs within six months, are especially problematic, as options for treatment and prevention are limited, often ineffective, and can be associated with untoward side effects. ${ }^{1}$ Antimicrobial resistance is now considered a major public health concern, and as such, efforts have been directed toward antimicrobial stewardship.

In this issue, Saatchi et al sought to examine the rates of rUTI cases and prescriptions in the presence of antimicrobial stewardship efforts in British Colombia over the course of a decade (2008-2018). Data for rUTI in adult women were collected from a provincial database, a physician billing system, and a consolidation file to combine antibiotic prescribing, diagnoses, and patient demographics. ${ }^{2}$

During the study period, the prevalence of rUTI cases declined by a whopping $59 \%$, with the steepest decline occurring in women aged $19-49$ years $(65 \%)$, and to a lesser degree, in women 80 years or older $(45 \%)$. Although not measured in this study, it is unlikely that established risk factors for rUTI (such as frequency of sexual intercourse, genitourinary syndrome of menopause, cystocele, and urinary incontinence) decreased during the study period. So what did change?

The authors surmise that decreasing rates of rUTI are most likely due to advances in clinical characterization of patients presenting with "rUTI." Clinical guidelines published during the study period, including those from the Canadian Urological Association (CUA) ${ }^{3}$ and the Infectious Disease Society of America (IDSA), 4,5 did certainly help clarify definitions and appropriate management of UTI, rUTI, and asymptomatic bacteriuria. An increased awareness that other genitourinary conditions can have UTI-like symptoms also likely decreased rUTI misdiagnosis. It is important to recognize that interstitial cystitis/bladder pain syndrome is one of several such conditions. Pelvic floor dysfunction, pelvic organ prolapse, and genitourinary syndrome of menopause are also associated with chronic UTI-like symptoms. It is imperative to thoroughly assess patients to determine if they are truly suffering from rUTI or possibly another condition with similar symptoms.

It is encouraging to see that, during the study period, there was a $34 \%$ decrease in overall prescriptions vs. cases of rUTI. This suggests a true decrease in antibiotic use from 2008-2018. Nitrofurantoin ascended to its rightful spot at as the first-line agent, and importantly, use of the highly resistant and adverse drug event-prone ciprofloxacin decreased by $86 \%$. However, before we pat ourselves on the back, ciprofloxacin did remain the second most prescribed antibiotic for rUTI. Most guidelines, including IDSA, ${ }^{5}$ suggest ciprofloxacin be used only as a last resort.

The lower level of both treatment and prophylactic antibiotics dispensed may be explained by greater antibiotic stewardship efforts (e.g., "Do Bugs Need Drugs?") but may also reflect a change in patient attitudes. We all have savvy patients who refuse antibiotics and demand alternatives. Clinicians now have better evidence supporting non-antibiotic alternatives for treatment (non-steroidal anti-inflammatory drugs) ${ }^{6}$ and prevention (increased hydration, cranberry, D-mannose, and immunomodulators). ${ }^{1}$ Low-dose antibiotic prophylaxis remains the most effective means of UTI prevention and, as the CUA/American Urological Association/Society of Urodynamics, Female Pelvic Medicine \& Urogenital Reconstruction rUTI guideline ${ }^{1}$ outlines, this continues to be a viable option in select patients. The ideal antibiotic, dose, and duration for this purpose remain unknown.

The limits of this study include potential misclassification bias due to the inclusion of the vague ICD-9 code ( $599=$ other disorders of urethra and urinary tract), lack of lab data, and lack of patient outcomes. 
This study of over 674785 rUTI cases and over 2 million rUTI-associated prescriptions shows a clear and encouraging decrease in rUTI prevalence and overall prescriptions from 2008-2018. This is likely multifactorial and seems, in part, due to antibiotic stewardship efforts.

Competing interests: The author does not report any competing personal or financial interests related to this work.

\section{References}

1. Anger J, Lee U, Ackerman AL, et al. Recurrent uncomplicated urinary tract infections in women: AUA/ CUA/SUFU guideline. J Urol 2019;202:282-9. https://doi.org/10.1097/JU.0000000000000296

2. Saatchi $A$, Yoo JW, Marra F. Outpatient prescribing and prophylactic antibiotic use for recurrent urinary tract infections in British Columbia, Canada. Can Urol Assoc J 2021;15:397-404. http://dx.doi. org/10.5489/cuaj.7162
3. Dason S, Dason JT, Kapoor A, et al. Guidelines for the diagnosis and management of recurrent urinary tract infection in women. Can Urol Assoc J 2013;5:316-22. https://doi.org/10.5489/cuai.687

4. Nicolle LE, Bradley $S$, Colgan R, et al. Infectious Diseases Society of America guidelines for the diagnosis and treatment of asymptomatic bacteriuria in adults. Clin Infect Dis 2005;40:643-54. https://doi.org/10.1086/427507

5. Gupta K, Hooton TM, Naber KG, et al. International clinical practice guidelines for the treatment of acute uncomplicated cystitis and pyelonephritis in women: A 2010 update by the Infectious Diseases Society of America and the European Society for Microbiology and Infectious Diseases. Clin Infect Dis 2011;52:e10320. https://doi.org/10.1093/cid/ciq257

6. Kronenberg A, Bütikofer L, Odutayo A, et al. Symptomatic treatment of uncomplicated lower urinary tract infections in the ambulatory setting: Randomized, double-blind trial. BMJ 2017;359:j4784. https://doi.org/10.1136/bmi.j4784

Correspondence: Dr. Duane R. Hickling, Division of Urology, Department of Surgery, The Ottawa Hospital, University of Ottawa, OHtawa, ON, Canada; duanehickling@gmail.com 